Article

\title{
Model for Elimination of Mixed Noise from MRI Heart Images
}

\author{
Vladyslav Shlykov ${ }^{1}$, Vitalii Kotovskyi ${ }^{2}$, Nikolaj Višniakov ${ }^{3, *}$ i] and Andžela Šešok ${ }^{4}$ \\ 1 Department of Biomedical Engineering, Igor Sikorsky Kyiv Polytechnic Institute, \\ National Technical University of Ukraine, Peremogy Ave. 37, 03224 Kiev, Ukraine; v.shlykov@kpi.ua \\ 2 Department of General Physics and Solid State Physics, Igor Sikorsky Kyiv Polytechnic Institute, \\ National Technical University of Ukraine, Peremogy Ave. 37, 03224 Kiev, Ukraine; kotovsk@kpi.ua \\ 3 Department of Mechanics and Material Engineering, Vilnius Gediminas Technical University, J. \\ Basanavičiaus Str. 28, 03221 Vilnius, Lithuania \\ 4 Department of Biomechanical Engineering, Vilnius Gediminas Technical University, J. Basanavičiaus Str. 28, \\ 03221 Vilnius, Lithuania; andzela.sesok@vgtu.lt \\ * Correspondence: nikolaj.visniakov@vgtu.lt; Tel.: +370-682-22-866
}

Received: 2 March 2020; Accepted: 7 July 2020; Published: 9 July 2020

check for updates

\begin{abstract}
A method for the preliminary processing of MRI images of the heart that allows for the elimination of fluctuation and impulse noise from useful signals is proposed. These types of noise are due to the regular geometric structure of the photoelectric elements of the MRI scanner matrix and the structure of the signal transmission channel. The aim of this work is to develop a comprehensive mathematical model for eliminating noise in the signal of an MRI scanner. In this work, mathematical models of linear and median filtering of impulse noise, fluctuation, and geometric noise are implemented. The mathematical models consist of the combined use of linear and median filters for recording MRI images of the heart. In the experiments, real MRI images of the heart from six patients with different diseases were used after noise was added to them. We were able to eliminate the impulse noise, geometric noise, and fluctuation noise in the MRI images by applying our filtering techniques. The filtering technique not only removed the noise, but also increased the contrast of the cancerous volumetric heterogeneous formations in the heart region.
\end{abstract}

Keywords: fluctuation noise; impulse noise; noise suppression; cardiac surgery; biological object

\section{Introduction}

Magnetic resonance imaging (MRI) images usually suffer from different types of noise, such as Gaussian noise, Poisson noise, speckle noise, etc. [1]. Currently, many particular methods for eliminating noise in MRI images have been developed for cases where the type of noise is known. The traditional approach to suppressing these types of noise is associated with the correction of the multiplicative and additive coefficients of the MRI scanner matrix [2]. This provides a sufficiently high uniformity of radiation intensity in the field of view. Examples of such methods are the full variation method and the median method of filtering one-dimensional signals [1-12], which are known to be effective approaches for eliminating Gaussian and Poisson noise [13-16]. Both types of noise (Poisson and Gaussian) have been well-studied, but their combination in a recorded signal [17], which is often present in biomedical signals and electron microscopy images, is also important [18,19]. However, these approaches are not without drawbacks, which are associated with the following:

- $\quad$ the need to use reference optical emitters (at least two);

- high uniformity requirements for the optical emitters in the field of view; 
- the need to use several reference emitters during the calibration process (if the dynamic range of the signals varies in space or time); and,

- the need to periodically repeat the calibration procedure (if the spectral composition of the transmission and signal processing channels changes over time).

In recent years, a number of studies have aimed to develop algorithms and practical application of mathematical models to suppress Gaussian and Poisson noise in information systems [20-23]. For example, the concept of full variation in eliminating Gaussian noise was first applied [13] based on the use of full variation in image processing tasks (Rudin-Osher-Fatem (ROF) model). This model is designed to eliminate Gaussian noise [13,14]; however, it can be used to eliminate other types of noise. Median filters are also quite often used as a software tool for image preprocessing in practice $[24,25]$.

Some MRI images are characterized by an increased noise level, or they contain noise or artifacts, which leads to an incorrect diagnosis. The quality of an MRI image depends on many factors: spatial resolution and image contrast, slice thickness, the reconstruction algorithm, physical processes in the measurement system, etc. The ambient temperature is one factor that affects the reliability of the obtained data. Fluctuations in temperature lead to an increase in the level of Gaussian noise in the MRI image and a decrease in measurement accuracy.

Developing methods and a means for eliminating noise and image artifacts in tomography is an urgent task. New ways to increase the signal-to-noise ratio have become possible thanks to the digitization of measurement results and the use of the DICOM (Digital Imaging and Communications in Medicine) 3.0 protocol. The use of this model to eliminate mixed noise from MRI images allows one to increase the signal-to-noise ratio and the quality of the images obtained, by eliminating Gaussian noise and by the further computer processing of images.

It is necessary to develop an approach for eliminating impulse noise and fluctuation noise in the temperature measurement channel in order to increase the reliability of diagnostics based on MRI images.

The relevance of the formulation of such a problem is determined by the software algorithm for suppressing geometric noise from the recorded images; this expands the possibilities for applying the results to other fields of biomedical research.

The aim of this work is to develop a comprehensive mathematical model for eliminating impulse noise, which is caused by various regular geometric structures of the photoelectric elements of the electronic matrix and the structure of the signal transmission channel, from MRI images.

\section{Methods}

The signal-to-noise ratio for an MRI image depends on a number of scanning parameters (pulse sequence, image matrix, resonance coil type) and substance properties (proton density, relaxation processes, diffusion). The use of various algorithms to reconstruct MRI images allows for one to adjust the contribution of a scan parameter to the intensity of the recorded signal to obtain optimal contrast between normal and altered tissues on an MRI image. It is possible to reduce random noise in MRI images by increasing the number of measurements, which leads to an increase in operating costs and an increase in the duration of a procedure. At the same time, reducing the scan time allows for one to eliminate measurement errors that are caused by a change in the position of the investigated object in space, but it requires the additional computer processing of images to eliminate random noise in MRI images.

The main methods for eliminating random noise in MRI images are rank and median filtering, Wiener filtering, correcting the initial k-space data, wavelet analysis and Fourier transform, and the statistical processing of experimental data. New ways to solve the problem of eliminating random noise in MRI images have become possible thanks to the use of the DICOM 3.0 protocol, which allows for efficient computer-based image correction due to the open graphic standard for digital data representation. Applying a model for eliminating mixed noise from images as an additional method of 
computer data processing allows us to improve the quality of MRI images of the internal structure of objects, which is an important scientific problem, while reducing the research time.

The following approximation system was used in order to simplify the software calculation algorithm and identify the main features of the studied approach:

1. the electronic matrix of MRI scanners have different shapes and geometric dimensions, however, for ease of implementation of the model, a square shape with piezoelectric elements of the same size and orientation was selected;

2. in addition to a useful image, the recorded data also contain fluctuation noise and pulsed flows of various intensities with a regular or chaotic structure (Gaussian and Poisson noise); and,

3. pulsed noise is understood to be a distortion of the recorded images by large pulsed emissions of arbitrary polarity and short duration, caused by both external pulsed electromagnetic interference and malfunctions of the system.

The model for eliminating the mixed noise from MRI images is a software algorithm for suppressing impulse noise, fluctuations, and geometric noise in the recorded signal. It is used for computer processing of MRI images in the format of DICOM files after an object is scanned.

The developed software algorithm for suppressing impulse noise, fluctuation, and geometric noise in the recorded image must have the property of universality.

\subsection{Model for Eliminating the Mixed Noise Based on Linear Signal Filtration}

The random process, which may appear as white noise $\xi(t)$, has a constant power spectral density $S_{\eta}(\omega)$ throughout the frequency domain, and its average power (dispersion) is infinite.

The model of a normal random process based on linear filtering is based on two principles of the theory of random processes:

1. the result of an arbitrary linear transformation of a Gaussian random process is a random Gaussian process; and,

2. the power spectral densities of random processes at the input $S_{\text {in }}(\omega)$ and output $S_{\text {out }}(\omega)$ of a linear filter with a frequency $H(i \omega)$ response are related by:

$$
\begin{gathered}
S_{\text {out }}(\omega)=|H(i \omega)|^{2} S_{\text {in }}(\omega) \\
H(i \omega)=\left\{\begin{array}{l}
1,\left|\omega-\omega^{\prime}\right| \leq \Delta \omega / 2 \\
0,\left|\omega-\omega^{\prime}\right|>\Delta \omega / 2
\end{array}\right.
\end{gathered}
$$

where $\omega^{\prime}$ and $\Delta \omega$ are the average frequency and filter bandwidth in the frequency band $\left[\omega^{\prime}-\Delta \omega / 2, \omega^{\prime}+\Delta \omega / 2\right]$.

To solve the linear filter modeling problem, it is necessary that the power spectral density $S_{\eta}(\omega)$ for a random process can be described by a fractional rational function of the variable $\omega$.

If white noise $\xi(t)$ enters at the input of the linear filter, then the spectral power density of the random process at the input will be equal to:

$$
S \eta(\omega)=\frac{N_{0}}{2},|\omega|<\infty
$$

Subsequently, the function of the spectral power density at the output of the linear filter can be represented in a fractional rational form:

$$
S_{\xi}(\omega)=\frac{b_{M} \omega^{2 M}+b_{M-1} \omega^{2(M-1)}+\cdots+b_{0}}{a_{N} \omega^{2 N}+a_{N-1} \omega^{2(N-1)}+\cdots+a_{0}}
$$

where $a_{N}$ and $b_{M}$ are polynomial roots in the numerator and denominator of the spectral density function for the power of white noise $S_{\eta}(\omega)$. 
The algorithm for modeling a Gaussian random process with specified spectral properties is based on transmitting white noise $\xi(t)$ with a single spectral density $S_{\eta}(\omega)=1$ through a linear filter whose frequency response corresponds to the expression (Figure 1):

$$
H(s)=\frac{F(s)}{G(s)}
$$

where

$$
\begin{gathered}
F(s)=\sqrt{\frac{b_{M}}{a_{N}}} \prod_{m=1}^{M}\left(s+f_{m}\right) \\
G(s)=\prod_{n=1}^{N}\left(s+g_{n}\right) \\
g_{n}=i a_{n}, n=\overline{1, N} \\
f_{m}=i \beta_{m}, m=\overline{1, M}
\end{gathered}
$$

where $a_{N}$ and $b_{M}$ are positive numbers in the power spectral density function of a random stationary process $H(s), s$ is a complex variable that corresponds to the actual signal frequency $\omega=i s$.
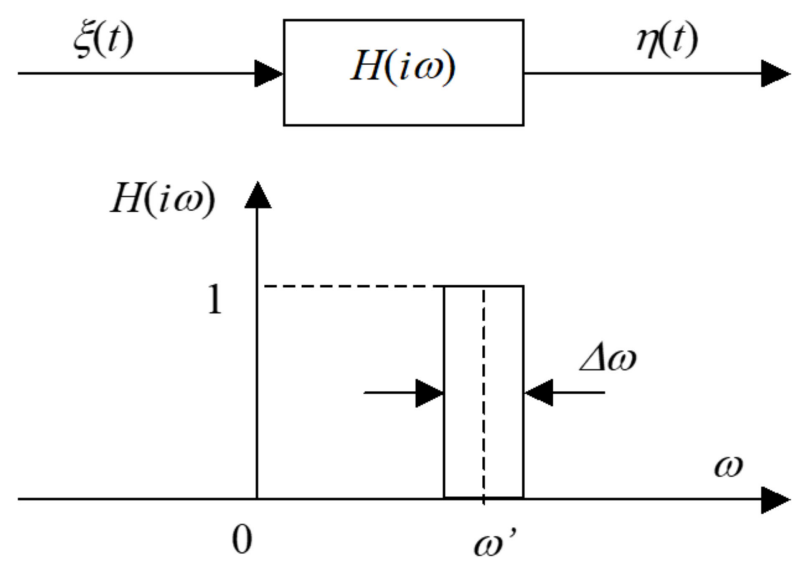

Figure 1. Functions of the power spectral density of a linear filter.

If white noise $\xi(t)$ with a single spectral density $N_{0} / 2=1$ is applied to the input of such a linear filter, then the signal will have a normal distribution at the output of the filter. Subsequently, the frequency characteristic of the filter $H(i \omega)=|H(i \omega)|$ and impulse characteristic of the linear filter take the following form:

$$
h(t)=\frac{1}{2 \pi} \int_{-\infty}^{+\infty}|H(i \omega)| e^{i \omega t} d \omega
$$

When a digital model of a signal with noise $\xi(t)$ is created, two general properties of white noise must be taken into account: the constant power spectral density $S_{\eta}(\omega)$ in the frequency domain and the statistical independence of time samples taken at arbitrary points in time $\Delta t$.

It is known that when a continuous signal $x(t)$ is sampled with a sampling period $\Delta t$, the spectral function of the sampled signal becomes periodic, with a period of $1 / \Delta t$.. Therefore, if the spectral power density $S_{\eta}(\omega)$ of the discrete white noise $\xi(t)$ is constant in the interval $[-0.5 / \Delta t ; 0.5 / \Delta t]$ and equal to $N_{0} / 2$, then it will be constant over the entire frequency domain. 
Subsequently, the variance of a random process with a uniform function of spectral power density $S_{\eta}(\omega)$ is determined by the formula:

$$
\sigma^{2}=\frac{1}{2 \pi} \int_{-0.5 / \Delta t}^{0.5 / \Delta t} \frac{N_{0}}{2} d \omega=\frac{1}{2 \pi \Delta t} \frac{N_{0}}{2}
$$

For a single spectral density $S_{\eta}(\omega)=N_{0} / 2=1$, we obtain that the discrete implementation of white noise $\xi(n)$ for the $N$ samples should be performed in accordance with the expression:

$$
\xi(n)=\frac{1}{\sqrt{2 \pi \Delta t}} x[n], n=\overline{1, N}
$$

where $x(n)$ are random independent numbers with a normal standard distribution (a Gaussian distribution with zero mathematical expectation and unit variance).

In modeling a linear filter in order to eliminate mixed types of noise based on a linear filtering method, its input $x(t)$ and output $y(t)$ signals are presented in the form of lattice functions $x[n]$ and $y[n]$, and are nonzero for time instants:

$$
t_{n}=n \Delta t, n=1,2, \ldots, N
$$

where $\Delta t$ is the sampling period of the signals.

Perfect equality of the input $x[n]=x(n \Delta t)$ and output signals $y[n]=y(n \Delta t)$ for the digital model of a linear filter is not achievable. In practice, approximate equality $y[n] \approx y(n \Delta t)$ is achieved with an error that has a normal distribution $S_{\text {out }}(\omega)$ with a dispersion equal to:

$$
\sigma=\sqrt{1 /(2 \pi \Delta t)}
$$

The application of this filtration model to an MRI image of the heart eliminates impulse noise as well as the geometric and fluctuation noise of the system (Figure 2).

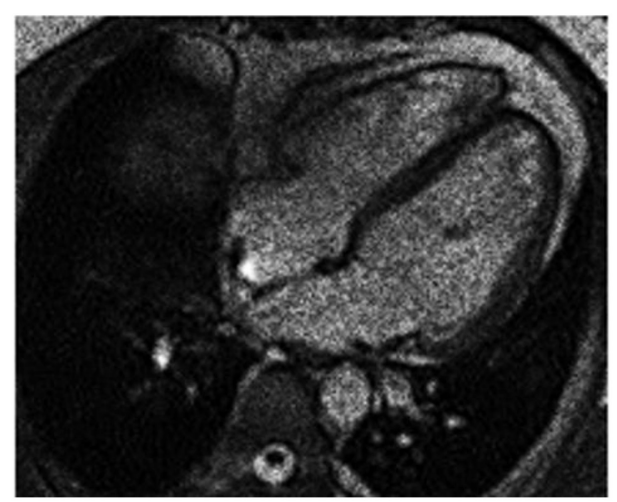

(a)

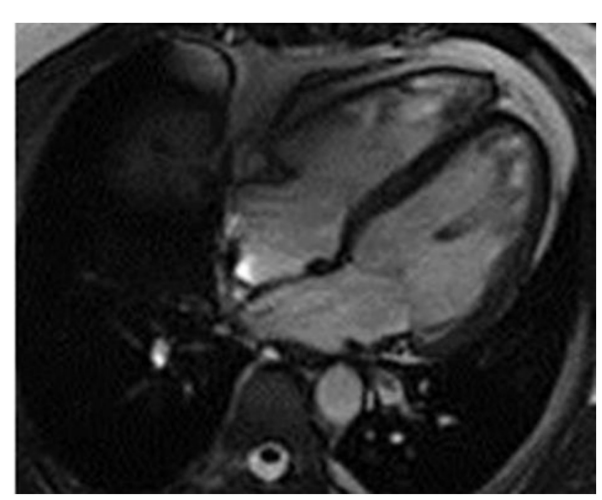

(b)

Figure 2. Heart MRI: (a) image with white noise and (b) denoised image.

Gaussian noise was added to the original MRI image in order to study the effectiveness of applying the model to eliminate mixed noise (Figure 2). The Gaussian noise was a simulation of the random noise in an MRI image in the DICOM format after a real biological object was scanned. It is assumed that the random noise in an MRI image of the heart is due to impulse noise and the geometric and fluctuation interference of the scanning system.

Thus, a digital model of a linear filter to eliminate mixed types of noise that represent a random process can be implemented while using a discrete Fourier transform by choosing the number of harmonics $M \approx 2 \pi / \Delta \omega$ and sampling frequency $\Delta t \geq M$ for the random process $\xi(n), n=\overline{1, N}$. 


\subsection{Model for Eliminating Mixed Noise Based on Median Signal Filtering}

Using a median filter to eliminate mixed noise is more effective than a linear filter. Linear filtering procedures are optimal for a uniform or Gaussian interference distribution, which implies a simplification of the noise structure in real signals.

Given the ranking of samples over time, median filters adequately suppress noise and interference, when the length is less than half the sliding window. A useful signal is a stable data sequence (in the case of a one-dimensional signal) or a data array (in the case of two-dimensional scanning) that does not change during median filtering.

Median filtering is implemented as an algorithm for the local processing of time samples in a sliding window, which includes a certain number of signal samples. For each position of the sliding window, the samples that are allocated in it are ranked according to increasing or decreasing values. The median in the ranked list is a group of samples positioned at the middle of the list.

It is assumed that the measured signal $x(t)$, in addition to the useful signal $s\left(t-\tau_{0}\right)$ and fluctuation noise $q(t)$, contains pulsed flows $g(t)$ of different intensities with a regular or chaotic structure:

$$
x(t)=s\left(t-\tau_{0}\right)+g(t)+q(t) .
$$

where $s\left(t-\tau_{0}\right)$ is a useful signal that has an unknown temporary position $\tau_{0} \in[0, T], q(t)$ is fluctuation noise with a regular or chaotic structure (Gaussian and Poisson noise), $g(t)=\sum_{k}(t-\tau k)$ are pulsed flows of various intensities with a regular or chaotic structure, and $\tau_{k}$ is the unknown temporal position of pulses.

According to probability theory, the distribution function $F(x)$ of the measured quantities $x=\operatorname{med}\left(x_{1}, \ldots, x_{n}\right)$ for large $n$ is approximately normal:

$$
F(x)=N\left(m_{t}, \sigma_{n}\right),
$$

where $m_{t}$ is a theoretical median determined from the condition $F\left(m_{t}\right)=0.5, \sigma_{n}$ is the distribution variance, and $n$ is the number of measurements of $x$.

In this case, the variance of the distribution of the median is determined as:

$$
\sigma_{n}^{2}=1 /\left(n 4 f^{2}\left(m_{t}\right)\right)
$$

where $f(x)$ is the probability density distribution of quantities $x$.

If the random variables $x$ are independent and equally distributed with the normal distribution $N(m, \sigma)$, then $m_{t}=m$, then the median dispersion formula for small odd values of $\mathrm{n}$ takes the form:

$$
\sigma_{\mathrm{g}}^{2} \approx \pi \sigma^{2} /(2 \mathrm{n}-2+\pi)
$$

If the function $f(x)$ is symmetric with respect to $m$, then the distribution of the medians will also be symmetric with respect to $m_{t}$.

Thus, the following formula is valid for determining the mathematical expectation:

$$
M\left\{\operatorname{med}\left(x_{1}, \ldots, x_{n}\right)\right\}=M\left\{x_{i}\right\}=m .
$$

The criterion, in accordance with which the size of the sliding window with central count $x_{r}$ is increased and the subsequent iteration is performed, takes the form:

$$
\max \left[S_{n}\left(x_{r}\right)\right]<S_{\text {threshold }}
$$

where $S_{n}\left(x_{r}\right)$ is the deviation of the amplitude of adjacent samples of the signal $x(t)$ that are located within the sliding window of size $n t$, relative to the amplitude of the central sample $x(r) ; S_{\text {threshold }}=[0, T]$ is the threshold coefficient of signal intensity. 
In Figure 3, an example of applying median filtering to a model signal $a_{k}$ is shown. It is composed of a deterministic signal $s_{k}$ and a random signal $q_{k}$ having a normal distribution with single pulse surges $\delta(t)$ of short duration.

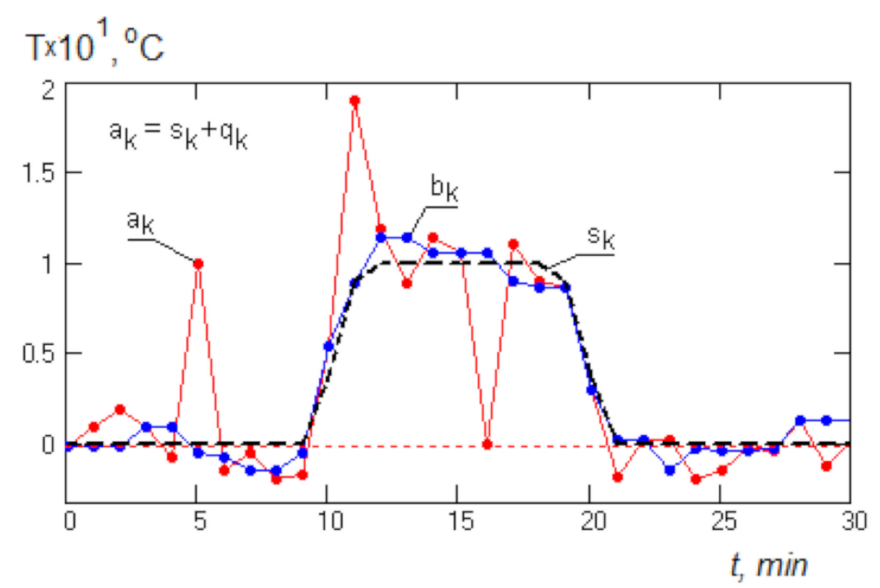

Figure 3. Example of applying median filtering to a model signal: $a_{k}$ is the model signal; $b_{\mathrm{k}}$ is the result of median filtering.

The results of calculating the median $m_{t}$ for the signal $x(t)$ are valid for both one-dimensional and two-dimensional filtering if the number of samples $n$ is equal to the number of points in the aperture of the median filter.

\section{Results and Discussion}

Clinical testing of models for eliminating noise in MRI images of the heart was performed to diagnose cancerous tumors in the heart, which form as a result of the progression of an underlying disease. The MRI images used in this work were obtained on a 1.5 Tesla MRI scanner using the closed-screen electrocardiological ECG protocol.

The MRI studies included a morphological review covering the whole heart with fast spin-echo sequences, sequential gradient echo sequences, and sequential precession echo sequences, which can significantly reduce the examination time.

Reliability analysis of the models was performed for several medical histories of tumor detection in patients, including those with lung cancer, with melanoma, with renal cell cancer, with sarcoma of the heart, with cardiac lymphoma, and with myxoma in the atrium [26].

The contour extraction method was used to identify cancerous areas that, after applying the model to eliminate mixed noise, had an increased contrast of volumetric heterogeneous formations in the region of the heart [27]. This method was developed by the authors when analyzing thermal images to assess the condition of the coronary vessels of the myocardium under cardiopulmonary bypass. The contours of the cancerous regions in the examples used for applying the model to eliminate mixed noise (Figures 4-9) are highlighted in yellow and they represent the visual boundary of the distribution of volumetric heterogeneous formations.

1. For a patient with a history of lung cancer: during MRI with contrast enhancement, the volumetric formation of a heterogeneous structure in the left ventricle was determined (Figure 4).

2. For a patient with a history of melanoma: during MRI with contrast enhancement, a volumetric formation adjacent to the atrial septum was visualized in the left atrium (Figure 5).

3. For a patient with renal cell carcinoma: during MRI with contrast enhancement in the right ventricle and interventricular septum, the volumetric formation of a heterogeneous structure in the form of infiltrated metastases was determined (Figure 6). 
4. For a patient with sarcoma of the heart: during MRI, a volumetric mass was determined in the lateral wall of the right atrium (Figure 7).

5. For a patient with heart and pericardial lymphoma: during MRI with contrast enhancement, the volumetric formation of a heterogeneous structure in the right atrium and thickened pericardium was determined (Figure 8).

6. For a patient with myxoma in the left atrium: during MRI with contrast enhancement in the left atrium, a hypodense mass formation, which causes mitral valve stenosis, was visualized (Figure 9).

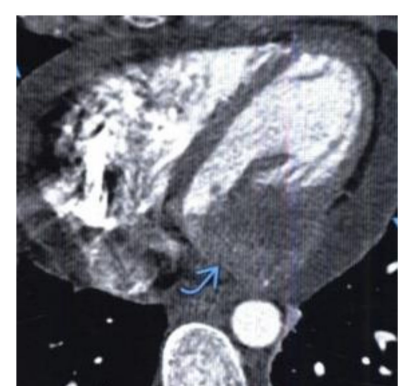

(a)

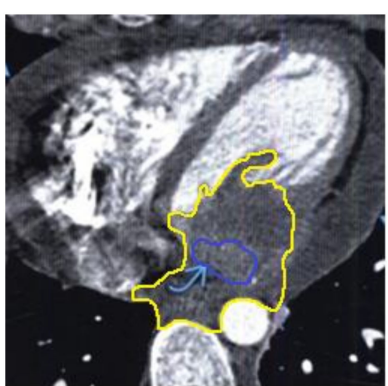

(b)

Figure 4. Heart MRI: (a) image with white noise and (b) denoised image with contrast enhancement of volumetric heterogeneous formation.

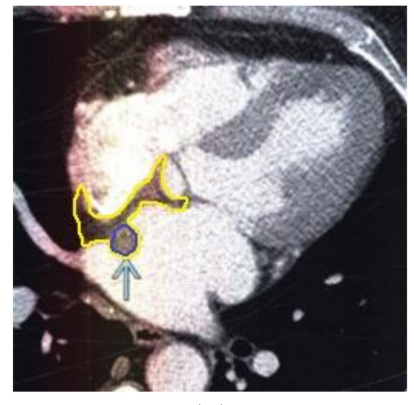

(a)

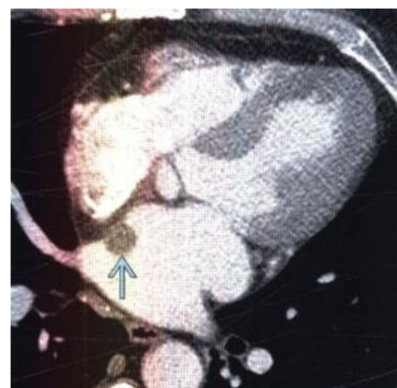

(b)

Figure 5. Heart MRI: (a) image with white noise and (b) denoised image with contrast enhancement of volume melanoma in the left atrium.

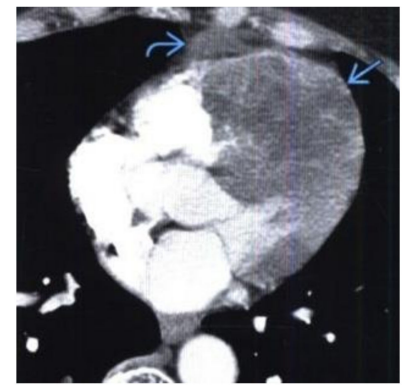

(a)

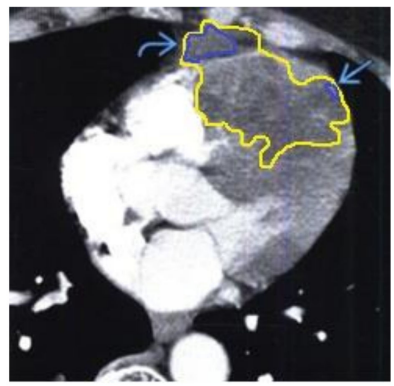

(b)

Figure 6. Heart MRI: (a) image with white noise and (b) denoised image with contrast enhancement of volumetric heterogeneous formation in the form of metastases. 


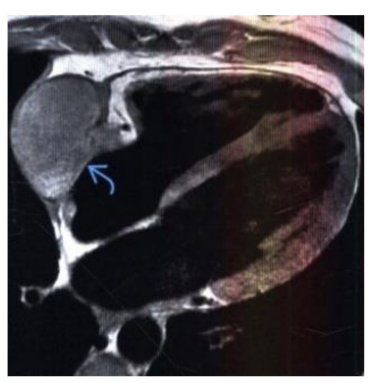

(a)

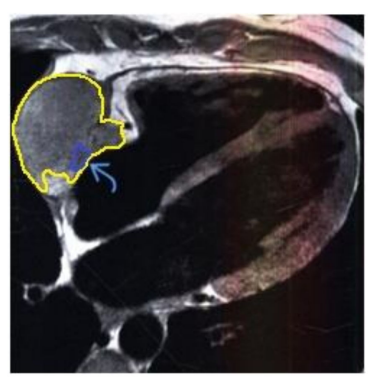

(b)

Figure 7. Heart MRI: (a) image with white noise and (b) denoised image with contrast enhancement of sarcoma in the right atrium.

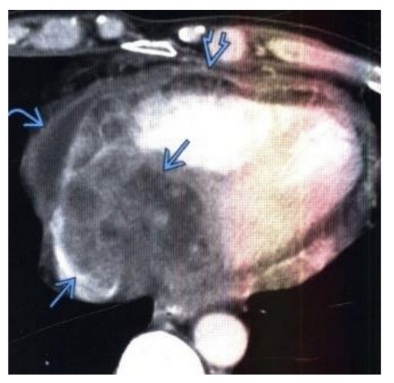

(a)

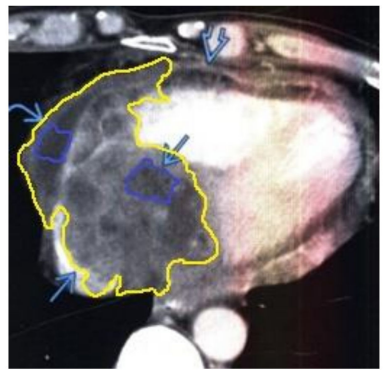

(b)

Figure 8. Heart MRI: (a) image with white noise and (b) denoised image with contrast enhancement of lymphoma in the right atrium.

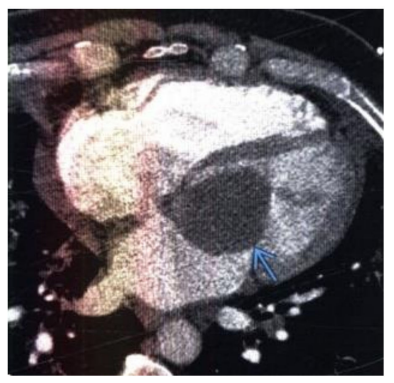

(a)

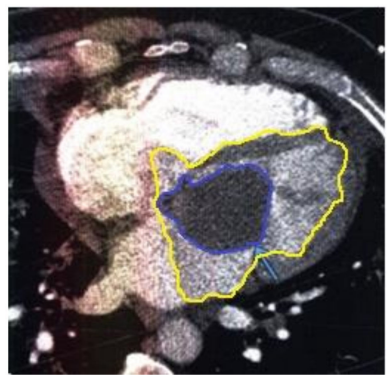

(b)

Figure 9. Heart MRI: (a) image with white noise and (b) denoised image with contrast enhancement of the myxoma in the left atrium.

A histological examination of all surgically removed emboli was performed to confirm the oncological diagnosis of these patients.

The quality of software processing was compared with other methods of noise elimination, for example, the ROF model, modified ROF model, and median filtering [24,25]. A mathematical model with a one-dimensional signal effectively eliminates combined Gaussian and Poisson noise, accounting for these types of noise.

As a result of research into and application of the filter model to eliminate impulse noise, and fluctuations and geometric noise from MRI images of the heart, it has become possible to detect and eliminate the random components of the image (noise), as well as reliably isolate and increase the contrast of cancerous volumetric heterogeneous formations in the heart region.

The block-match and three-dimensional (3D) filtering (BM3D) filter [28] mentioned in the review [21] has shown good results in many areas, including MRI [29]. BM3D utilizes the notion that natural images have self-spatial similarities. First, the algorithm searches for patches in the image that are similar to the intensities of the patch that has to be denoised. This method builds a 3D matrix where the first two dimensions are the size of the patch while the third dimension is the aggregated 
patches. BM3D applies a 3D unitary sparsifying transform. This transform space is then thresholded and Wiener filtered in order to achieve coefficient shrinkage to remove high frequency noises [29]. Our proposed method of processing MRI images is an alternative to existing ones. The presented model for eliminating the mixed noise from MRI images is a software algorithm for suppressing impulse noise, fluctuations, and geometric noise in the recorded signal. It is used for computer processing of MRI images in the format of DICOM files after an object is scanned. To identify cancerous areas, the contour extraction method that was developed by the authors was used. An increase in the contrast of cancerous volumetric heterogeneous formations in the region of the heart due to an increase in the signal-to-noise ratio by $10 \%$ after processing the MRI image in the DICOM file is technically sufficient to highlight the contours of the formation. The dynamics of narrowing or expanding the contours of the cancerous area during sequential studies allows for us to provide a clinical prognosis of the stage of the disease.

\section{Conclusions}

Mathematical models of linear and median filtering of impulse, fluctuation, and geometric noise were implemented, which consisted of the combined use of linear and median filters for registering MRI images of the heart. The main types of noise that were eliminated by the implemented filtering models were thermal noise caused by thermal fluctuations in the image receiver.

Thus, using our model based on the method of median filtering of signals, we were able to eliminate impulse noise, geometric noise, and fluctuation noise in MRI images. The filtering algorithm allowed not only a reduction noise, but also an increase in the contrast of cancerous volumetric heterogeneous formations in the region of the heart.

The use of a mathematical model to eliminate mixed noise in the processing of data that were obtained during the preliminary processing of MRI images allowed for an increase the reliability of the clinical diagnosis of oncological volumetric heterogeneous formations in the heart.

Author Contributions: Conceptualization, methodology, project administration by V.K.; software, validation, formal analysis, investigation by V.S.; resources, review and editing, supervision, funding acquisition by N.V.; data curation, visualization, writing, original draft preparation by A.Š. All authors have read and agreed to the published version of the manuscript.

Funding: This research received no external funding.

Conflicts of Interest: The authors declare no conflicts of interest.

\section{References}

1. Halefoğlu, A.M. High-Resolution Neuroimaging. Basic Physical Principles and Clinical Applications; Books on Demand: Norderstedt, Germany, 2018. [CrossRef]

2. Chambolle, A.; Caselles, V.; Cremers, D.; Novaga, M.; Pock, T. An introduction to total variation for image analysis. Theor. Found. Numer. Methods Sparse Recovery. 2010, 9, 263-340. [CrossRef]

3. Chan, T.F.; Shen, J. Image Processing and Analysis: Variational, PDE, Wavelet, and Stochastic Methods, 1st ed.; SIAM: Philadelphia, PA, USA, 2005; p. 186.

4. Burger, M.; Mennucci, A.C.G.; Osher, S.; Rumpf, M. Level Set and PDE-Based Reconstruction Methods in Imaging; Springer: Cham, Switzerland, 2008; p. 319.

5. $\mathrm{Xu}, \mathrm{J} . ; \mathrm{Feng}, \mathrm{X} . ; \mathrm{Hao}, \mathrm{Y}$. A coupled variational model for image denoising using a duality strategy and split Bregman. Multidimens. Syst. Signal Process. 2014, 25, 83-94. [CrossRef]

6. Rankovic, N.; Tuba, M. Improved adaptive median filter for denoising ultrasound images. Advances Comput. Sci. 2012, 12, 169-174.

7. Lysaker, M.; Tai, X. Iterative image restoration combining total variation minimization and a second-order functional. Int. J. Comput. Vis. 2006, 66, 5-18. [CrossRef]

8. Li, F.; Shen, C.; Pi, L. A new diffusion-based variational model for image denoising and segmentation. J. Math. Imaging Vis. 2006, 26, 115-125. [CrossRef] 
9. Zhu, Y.; Zhao, M.; Zhao, Y.; Li, H.; Zhang, P. Noise reduction with low-dose CT data based on a modified ROF model. Opt. Express 2012, 20, 17987-18004. [CrossRef] [PubMed]

10. Tran, M.P.; Peteri, R.; Bergounioux, M. Denoising 3D medical images using a second order variational model and wavelet shrinkage. Lecture Notes in Computer Science. In Proceedings of the Image Analysis and Recognition, Aveiro, Portugal, 25-27 June 2012; pp. 138-145.

11. Getreuer, P. Rudin-Osher-Fatemi total variation denoising using split Bregman. Image Process. Line 2012, 2, 74-95. [CrossRef]

12. Scherzer, O. Handbook of Mathematical Methods in Imaging; Springer: New York, NY, USA, 2011; p. 1607. [CrossRef]

13. Rudin, L.I.; Osher, S.; Fatemi, E. Nonlinear total variation-based noise removal algorithms. Phys. D Nonlinear Phenom. 1992, 60, 259-268. [CrossRef]

14. Chen, K. Introduction to variational image processing models and application. Int. J. Comput. Math. 2013, 90, 1-8. [CrossRef]

15. Orea-Flores, I.Y.; Gallegos-Funes, F.J.; Arellano-Reynoso, A. Local complexity estimation-based filtering method in wavelet domain for magnetic resonance imaging denoising. Entropy 2019, 21, 401. [CrossRef]

16. Le, T.; Chartrand, R.; Asaki, T.J. A variational approach to reconstructing images corrupted by Poisson noise. J. Math. Imaging Vis. 2007, 27, 257-263. [CrossRef]

17. Luisier, F.; Blu, T.; Unser, M. Image denoising in mixed Poisson-Gaussian noise. IEEE Trans.Image Process. 2011, 20, 696-708. [CrossRef] [PubMed]

18. Jezierska, A.; Chaux, C.; Pesquet, J.C.; Talbot, H.; Engler, G. An EM approach for Poisson-Gaussian noise modelling. In Proceedings of the 19th European Signal Processing Conference EUSIPCO, Barcelona, Spain, 29 August-2 September 2011; pp. 13-30.

19. Jezierska, A.; Talbot, H.; Chaux, C.; Pesquet, J.C.; Engler, G. Poisson-Gaussian noise parameter estimation in fluorescence microscopy imaging. In Proceedings of the 9th International Symposium on Biomedical Imaging, Barcelona, Spain, 2-5 May 2012; pp. 1663-1666. [CrossRef]

20. Wang, Z.; Bovik, A. Modern image quality assessment. Synth. Lect. Image Video Multimedia Process. 2006, 2, 156. [CrossRef]

21. Fan, L.; Zhang, F.; Fan, H.; Zhang, C. Brief review of image denoising techniques. Vis. Comput. Ind. Biomed. Art 2019, 2-7. [CrossRef] [PubMed]

22. Milanfar, P. A tour of modern image filtering: New insights and methods, both practical and theoretical. IEEE Signal. Process. Mag. 2013, 30, 106-128. [CrossRef]

23. Buades, A.; Coll, B.; Morel, J.M. A non-local algorithm for image denoising. In Proceedings of the IEEE Computer Society Conference on Computer Vision and Pattern Recognition, San Diego, CA, USA, 20-25 June 2005; pp. 60-65. [CrossRef]

24. Pitas, I.; Venetsanopoulos, A.N. Nonlinear Digital Filters: Principles and Applications; Springer Nature: Cham, Switzerland, 1990; p. 392. [CrossRef]

25. Wang, C.; Li, T. An improved adaptive median filter for image denoising. In Proceedings of the International Conference on Computers in Education ICCE, Singapore, 26-30 November 2012; pp. 393-398. [CrossRef]

26. Russian Cancer Research Center NN Blokhin. Radiation Diagnostics: Examples of the Diagnosis of Heart Tumors by X-Ray, CT, MRI. Access Mode. Available online: https://meduniver.com/Medical/luchevaia_ diagnostika/primeri_diagnostiki_opuxolei_serdca.html (accessed on 11 September 2019).

27. Shlykov, V.; Kotovskyi, V.; Višniakov, N.; Šešok, A. The IR-thermal imaging method for evaluation of the status of myocardial coronary vessels under the condition of artificial blood circulation. Technol. Health Care 2018, 27, 571-576. [CrossRef] [PubMed]

28. BM3D Matlab Package. Available online: http://www.cs.tut.fi/ \{\}foi/GCF-BM3D/bm3d_matlab_package_3.0. 5.zip (accessed on 16 February 2020).

29. Chaudhari, A. Denoising for Magnetic Resonance Imaging. Available online: http://stanford.edu/class/ee367/ Winter2016/Chaudhari_Report.pdf (accessed on 17 February 2020).

(C) 2020 by the authors. Licensee MDPI, Basel, Switzerland. This article is an open access article distributed under the terms and conditions of the Creative Commons Attribution (CC BY) license (http://creativecommons.org/licenses/by/4.0/). 